\title{
Protocolo GUADALMED (PRECE).
}

\author{
Pablo Jáimez-Cuéllar ${ }^{*}$, Soledad Vivas ${ }^{6}$, Núria Bonada ${ }^{4}$, Santiago Robles ${ }^{3}$, \\ Andrés Mellado ${ }^{5}$, Maruxa Álvarez ${ }^{2}$, Juan Avilés ${ }^{3}$, Jesús Casas ${ }^{6}$, Manuel Ortega ${ }^{6}$, \\ Isabel Pardo ${ }^{2}$, Narcís Prat ${ }^{4}$, Maria Rieradevall ${ }^{4}$, Carmen Elisa Sáinz-Cantero ${ }^{1}$, \\ Antonino Sánchez-Ortega ${ }^{1}, \mathrm{M}^{\mathrm{a}}$ Luisa Suárez ${ }^{5}$, Manuel Toro ${ }^{3}, \mathrm{M}^{\mathrm{a}}$ Rosario Vidal-Abarca ${ }^{3}$, \\ Carmen Zamora-Muñoz ${ }^{1}$ y Javier Alba-Tercedor ${ }^{1 \#}$
}

${ }^{1}$ Departamento de Biología Animal y Ecología. Universidad de Granada. Campus Universitario de Fuentenueva. 18071 Granada.

${ }^{2}$ Área de Ecología. Universidad de Vigo. Campus Lagoas-Marcosende. 36200 Vigo.

${ }^{3}$ CEDEX. División de Ecología de los Sistemas Acuáticos Continentales. Paseo Bajo Virgen del Puerto, 3. 28005 Madrid.

${ }^{4}$ Departament d'Ecologia. Universitat de Barcelona. Diagonal, 645. 08028 Barcelona.

${ }^{5}$ Departamento de Ecología e Hidrología. Universidad de Murcia. Campus de Espinardo. 30100 Murcia.

${ }^{6}$ Departamento de Biología Vegetal y Ecología. Universidad de Almería. Cañada de San Urbano, s/n. 04120 Almería.

\section{RESUMEN}

En el seno del proyecto GUADALMED se ha desarrollado un Protocolo Rápido de Evaluación de la Calidad Ecológica (PRECE) válido para aplicar a los ríos Mediterráneos. En él se proporciona la metodología necesaria para evaluar la calidad de la vegetación de ribera mediante el índice QBR, la calidad biológica del agua mediante el índice IBMWP (nueva versión modificada del BMWP'), y la heterogeneidad del hábitat fluvial mediante el índice IHF. Además se estandariza la medida del caudal y la toma de muestras para los análisis de físico-química de las aguas.

Palabras clave: Calidad Ecológica, Protocolo, PRECE, QBR, IBMWP, IHF, Ríos mediterráneos, Directiva Marco del Agua

\begin{abstract}
A rapid protocol for Evaluation of the Ecological Status of Mediterranean rivers (RBP) has been designed within the GUADALMED project. This protocol provides the necessary methodology to determine the value of three indices: the $Q B R$ index (to evaluate riparian quality), the IBMWP index (former BMWP' for the assessment of biological quality of water), and the IHF index (physical habitat). The protocol also includes methods for measuring river discharge and physicochemical variables of water.
\end{abstract}

Keywords: Ecological Status, protocol, RBP, QBR, IBMWP, IHF, Mediterranean rivers, Water Frame Directive

\section{INTRODUCCIÓN}

La Directiva Marco del Agua (DMA) (D.O.C.E., 2000) prevé que los países miembros determinen el estado ecológico de sus aguas continentales basándose en criterios biológicos. La escala de calidades biológicas medidas deberá ser objeto de una intercalibración entre los sistemas emple- ados por los Estados miembros que, por lo que concierne a los ríos mediterráneos, es uno de los objetivos fundamentales del proyecto GUADALMED. El objetivo del proyecto GUADALMED es realizar un estudio en detalle de varios ríos mediterráneos españoles, que por las características intrínsecas de estos ríos (sequías, avenidas) pueden presentar dificultades en la determina-

* Dirección actual: Hydraena S.L.L. Nenúfares, 8. 18170 Jun (Granada).

\# Persona de contacto

Limnetica 21(3-4): 187-204 (2002)

(C) Asociación Española de Limnología, Madrid. Spain. ISSN: 0213-8409 
ción de su estado ecológico, lo cual significa problemas metodológicos que resolver antes de la aplicación de la DMA. El objetivo final es la definición de un índice integrado para clasificar los ríos de acuerdo con su Estado Ecológico aplicable a todos los ríos Mediterráneos.

El Protocolo Rápido de Evaluación de la Calidad Ecológica (PRECE) desarrollado dentro del proyecto GUADALMED incluye una síntesis de metodologías usadas para la aplicación de diversos índices de calidad. La ventaja de seguir un protocolo establecido en la aplicación de las diferentes metodologías es que los datos analíticos obtenidos puedan ser comparables entre diferentes grupos de trabajo. Estos aspectos son muy importantes en proyectos como el que se ha llevado a cabo, donde participan numerosos grupos de trabajo diferentes. Algunos índices utilizados ya existían antes de empezar este estudio, como son el índice BMWP' de calidad biológica del río (AlbaTercedor \& Sánchez-Ortega, 1988; Alba-Tercedor, 1996; Alba-Tercedor \& Pujante, 2000), que ha sido modificado hasta convertirse en el nuevo IBMWP (Alba-Tercedor et al., en este volumen) y el índice QBR, de evaluación del estado del bosque de ribera (Munné et al., 1998; Suárez-Alonso \& Vidal-Abarca, 2000; Munné et al., 2003).
Mientras que el IHF, de caracterización del hábitat fluvial (Pardo et al. ver este volumen) se ha desarrollado expresamente para este proyecto.

Para realizar este protocolo hemos usado las pautas seguidas en los protocolos de la Agencia Americana de Protección Ambiental (Plafkin et al., 1989; Barbour et al., 1999), en los que se inspiró el protocolo ECOSTRIMED desarrollado por miembros del proyecto GUADALMED (Prat et al., 2000). En todo momento se ha tenido en cuenta el hecho de que los distintos métodos fueran fáciles de utilizar, baratos y de amplia aplicabilidad sin perder su eficacia (Resh \& Jackson, 1993; Resh et al., 1995), de modo que sirvieran de base para el estudio del estado ecológico de los ríos mediterráneos permitiendo la aplicación de la DMA en estos ecosistemas. Así pues se trata de un RBP (Rapid Bioassesment Portocol) (Wright et al., 1984; Davies, 1994; Barbour et al., 1999) al que 1lamaremos PRECE (Protocolos Rápidos de Evaluación de la Calidad Ecológica).

Las recomendaciones dentro del capítulo de la toma de muestras para las medidas de físicoquímica son una recopilación de los protocolos propuestos en las normas UNE (AENOR, 1997) (Tabla 1), tanto en sus procedimientos

Tabla 1. Versiones of iciales adoptadas por España de las Normas Europeas UNE utilizadas para la redacción de los procedimientos citados. Se incluye la Norma Internacional ISO correspondiente. Official versions of the European Guidelines UNE adopted by Spain and used in the procedures cited. The associated international guideline ISO is also included.

\begin{tabular}{|c|c|c|c|}
\hline Norma UNE española & Título & Norma europea & Norma internacional ISO \\
\hline UNE-EN 25667-1: 1995 & $\begin{array}{l}\text { Calidad del agua. Muestreo. } \\
\text { Parte 1: Guía para el diseño de } \\
\text { los programas de muestreo. }\end{array}$ & EN 25667-1: 1993 & ISO 5667-1:1980 \\
\hline UNE-EN 25667-2: 1995 & $\begin{array}{l}\text { Calidad del agua. Muestreo. } \\
\text { Parte 2: Guía para las técnicas } \\
\text { de muestreo. }\end{array}$ & EN 25667-2: 1993 & ISO 5667-2:1991 \\
\hline UNE-EN ISO 25667-3: 1996 & $\begin{array}{l}\text { Calidad del agua. Muestreo. } \\
\text { Parte 3: Guía para la conservación } \\
\text { y manipulación de las muestras. }\end{array}$ & EN ISO 25667-3: 1995 & ISO 5667-2:1994 \\
\hline UNE-EN 27828: 1995 & $\begin{array}{l}\text { Calidad del agua. Métodos } \\
\text { de muestreo biológico. Guía } \\
\text { para el muestreo manual de } \\
\text { macroinvertebrados bénticos }\end{array}$ & EN 27828: 1995 & ISO 7828: 1985 \\
\hline UNE-EN 28265: 1995 & $\begin{array}{l}\text { Calidad del agua. Concepción } \\
\text { y utilización de los muestreadores } \\
\text { de macroinvertebrados bénticos } \\
\text { sobre sustrato rocoso en aguas dulces } \\
\text { poco profundas }\end{array}$ & EN 28265: 1995 & ISO 8265: 1988 \\
\hline
\end{tabular}


generales de técnicas de muestreo, como en los que se refieren a parámetros específicos. Alguna de las sugerencias de muestreo, conservación de muestras, etc. se han recopilado de los Métodos Normalizados (APHA, 1992). Otras recomendaciones se han redactado teniendo en cuenta la experiencia de los grupos de trabajo GUADALMED y las propias necesidades surgidas dentro del proyecto.

Los diferentes métodos han sido aplicados a 157 puntos de muestreo repartidos en 12 cuencas hidrográficas a lo largo de toda la cuenca mediterránea, 10 de ellas situadas en la vertiente mediterránea de la Península Ibérica y dos en las Islas Baleares.

\section{Pautas generales}

Se deben seguir los siguientes pasos:

1. Seleccionar la estación de muestreo. Los criterios de selección pueden encontrarse en Bonada et al., en este volumen.

2. Identificación del punto de muestreo (nombre, código, fecha, hora)

3. Toma de muestras

3.1. Fisico-química del agua (protocolo 1)

3.2. Caudal (protocolo 1)

3.3. Hábitat (protocolo 2)

3.4. Macroinvertebrados (protocolo 3 )

3.5. Vegetación de ribera (protocolo 4)

\section{MATERIAL NECESARIO PARA EL MUESTREO}

\section{ANTES DE COMENZAR:}

- Permiso de muestreo

- Mapas

- Cámara de fotos

- Fichas de campo

- Botas de agua

- Guantes de goma

- Trapo o toalla

\section{MUESTREO DE LA FISICOQUÍMICA:}

- Termómetro

- pH-metro y soluciones tampón para calibrar el pH-metro

- Conductímetro

- Oxímetro o Botellas y reactivos Winkler

- Membrana de repuesto para el oxímetro

- Botes y botellas para la muestra de agua

- Cloroformo (si se fija la muestra de agua con cloroformo)

- Cuentagotas

- Nevera con bloques helados

- Pilas de repuesto para todos los aparatos

- Frascos lavadores con agua destilada y rollo de papel de celulosa (para enjuague y limpieza de electrodos)

\section{Caudal:}

- Cinta métrica de al menos 10 metros y metro rígido

- Correntímetro

\section{PARA ETIQUETAR:}

- Rotuladores indelebles

- Lápices y sacapuntas

- Etiquetas de papel y cinta aislante para botes y botellas

\section{PARA EL MUESTREO BIOLÓGICO:}

\section{Macroinvertebrados:}

- Redes de 300 micras (unas de mango corto y otras de mango largo)*

- Pinzas entomológicas y/o aspirador entomológico

- Bateas blancas de plástico (mínimo 30 x $20 \mathrm{~cm}$ )

- Cuentahilos o lupa (ayuda identificación organismos)

- Viales de plástico herméticos

- Alcohol de $96^{\circ}$

- Formol $40 \%$

Estaciones de referencia:

- Bolsas de plástico o botes grandes para la muestra de macroinvertebrados de las estaciones de referencia

Vegetación de ribera:

- Guía de árboles y arbustos

* Con corrientes fuertes, utilizar redes de 500-1000 $\mu \mathrm{m}$. 


\section{PROTOCOLO 1: DE TOMA DE MUESTRAS PARA LA REALIZACIÓN DE ANÁLISIS FÍSICO-QUÍMICOS}

\section{Pasos a seguir}

\section{Seleccionar el área de toma de muestra}

Para que el muestreo sea lo más representativo posible se debe realizar la toma de muestras en puntos que representen el tipo de hábitat de estudio. Seleccionar zonas donde el agua esté bien mezclada (zonas centrales), evitando tomar agua superficial, rebosaderos de los embalses, confluencias de ríos poco importantes, lugares de pequeños vertidos, etc. ya que sólo tienen efectos muy localizados en la química del agua de ese tramo, y evaluarían incorrectamente el estado del río y las características del agua en el tramo de estudio. Muestrear antes del comienzo de los trabajos que puedan modificar las características del agua (en especial el muestreo biológico). Otra posibilidad es realizarlo posteriormente, aguas arriba de la zona perturbada.

\section{Medidas instantáneas con equipos portátiles (Tabla 2)}

La temperatura, el pH o los gases disueltos cambian de manera significativa en cuestión de minutos y por ello hay que medirlos en el mismo momento del muestreo con ayuda de los electrodos de campo.

Antes del muestreo es imprescindible leer minuciosamente las instrucciones de uso de los aparatos que se vayan a utilizar, comprobando el buen estado de los mismos (lo que incluye revisión y limpieza).

Controlar la temperatura de referencia o de calibrado del aparato. La temperatura normalmente utilizada suele ser $25{ }^{\circ} \mathrm{C}, \mathrm{y}$ este dato deberá incluirse en la hoja de campo que contenga las medidas realizadas. Si la calibración se realiza con líquidos tampón, como el caso del pH, los rangos esperados de esta medida serán los que utilicemos para elegir las soluciones tampón calibradoras. Estas se deben utilizar a la misma temperatura a la que se encuentre el agua a medir. Esto se consigue sumergiendo los tampones unos minutos en el río hasta que la temperatura de los mismos se iguale con la del agua del río.

Estas medidas directas se deben realizar, en la medida de lo posible, en el mismo río sin necesidad de recoger el agua en un recipiente ya que la manipulación de la muestra puede modificar la solubilidad de los gases disueltos.

Los electrodos, una vez sumergidos, no deben entrar en contacto con macrófitos o algas, o quedar enterrados en el sedimento ya que esto puede desvirtuar la medida real.

\section{Medidas de laboratorio: toma de muestras. \\ Traslado y conservación (Tabla 2)}

Algunos análisis químicos se ven mucho más afectados que otros por los efectos de la conservación de las muestras. Con una correcta técnica de conservación y transporte de las muestras hasta el laboratorio se consigue retrasar los cambios químicos y biológicos que se producen inevitablemente después de la toma.

Los recipientes que contendrán la muestra de agua y la transportarán hasta su análisis definitivo deben estar totalmente limpios. Dependiendo del procedimiento a seguir (normalmente en los análisis de fósforo en los que no se utilice un recipiente estéril y que no se vayan a realizar inmediatamente), se deberá realizar un lavado meticuloso del recipiente con ácido diluido. Dependiendo del analito, se exige que los recipientes dónde se almacene la muestra sean de un tipo de material u otro (Tabla 3). La elección de un recipiente apropiado y su preparación puede ser de la mayor importancia a la hora de realizar análisis químicos.

Tabla 2. Parámetros físico-químicos determinados en el proyecto GUADALMED. Physical and chemical variables measured in the Guadalmed project.

\section{MEDIDAS INSTANTÁNEAS \\ EN EL PUNTO DE MUESTREO}

\section{$\mathrm{T}^{\mathrm{a}}\left({ }^{\mathrm{o}} \mathrm{C}\right)$}

Conductividad $\left(\mu \mathrm{S} / \mathrm{cm}, 25^{\circ} \mathrm{C}\right)$

Oxígeno disuelto ( $\mathrm{mg} / \mathrm{l}$ y \% saturación)

$\mathrm{PH}$

\section{MEDIDAS Y ANÁLISIS POSTERIORES}

\author{
Alcalinidad \\ N-Nitratos $\left(\mathrm{mg} / \mathrm{l} \mathrm{N}-\mathrm{NO}_{3}\right)$ \\ N-Nitritos (mg/l N-NO $\left.{ }_{2}\right)$ \\ Amonio (mg/ $\left.1 \mathrm{NH}_{4}{ }^{+}\right)$ \\ Ortofosfato ${ }^{1}\left(\mathrm{mg} / 1 \mathrm{P}-\mathrm{PO}_{4}\right)$ \\ Cloruros $\left(\mathrm{mg} / \mathrm{l} \mathrm{Cl}^{-}\right)$ \\ Sulfatos (mg/l SO$\left.{ }_{4}^{2-}\right)$ \\ Sólidos en suspensión (mg/l)
}


Antes de la toma de la muestra todos los recipientes deben limpiarse concienzudamente con el agua del río, enjuagando al menos 6 veces.

Los recipientes deben ser llenados completamente hasta su parte superior y taparlos de manera que se impida que quede aire encima de la muestra y la formación de burbujas. Esto limita la interacción con la fase gaseosa y la agitación durante el transporte y conseguiremos que quede la menor cantidad de aire en el recipiente que pueda interferir con el analito y desvirtuar la medición (muy importante en casos como la medida de la alcalinidad). Éste será un procedimiento válido en todos los casos excepto en los que vayan a fijarse para su conservación. En este caso se dejará un poco de volumen en el recipiente para añadir la sustancia fijadora, igual que en el caso de que el contenido se vaya a congelar.
Las principales recomendaciones para el traslado y conservación de las muestras, según el analito que queramos medir, se describen en la Tabla 3.

En general no se pueden dar reglas absolutas y fijas para la conservación, la duración de la conservación, la naturaleza del recipiente y la eficacia de los procesos de conservación. Esto se debe a que dependen, no sólo de los constituyentes que han de analizarse y de sus niveles sino también de la naturaleza de la muestra. Por tanto, el contenido de la Tabla 3 debe considerarse únicamente como sugerencias razonables. En cada caso concreto y para cada método de análisis a utilizar se deberá comprobar si las indicaciones de la tabla son las apropiadas. Como norma general se deberá evitar la congelación de las muestras como forma de conservación de las mismas. Si está es la única forma posible de mantenerlas hasta su análisis, se

Tabla 3. Sugerencia de recipientes, técnicas de conservación y tiempos máximos de almacenamiento. Los datos de la tabla están tomados de AENOR (1997) y APHA (1992). Plástico (P) (e.g., Polietileno o PVC); Vidrio (V); Vidrio Borosilicatado (VB). La conservación de la muestra debe ser realizada inmediatamente después de su recolección. Las muestras deben ser analizadas tan pronto como sea posible. Los tiempos que aquí se sugieren son tiempos máximos considerados válidos para que la muestra permanezca estable. ${ }^{1}$ Fósforo reactivo soluble. Suggestions about containers, preservation techniques and maximum storage time. Data were obtained from AENOR (1997) and APHA (1992). Plastic (P) (e.g., Polyethylene or PVC); Glass (V); Borosilicate glass (VB). Samples must be preserved immediately after collection. Samples must be analysed as soon as possible. Times presented here are considered valid for maintaining an essentially unaltered sample. ${ }^{~}$ Soluble reactive phosphorus.

\begin{tabular}{|c|c|c|c|c|}
\hline Parámetro & $\begin{array}{l}\text { Material } \\
\text { del } \\
\text { recipiente }\end{array}$ & Conservación & $\begin{array}{l}\text { Tiempo } \\
\text { máximo de } \\
\text { almacenamiento }\end{array}$ & Observaciones \\
\hline Alcalinidad & $\mathrm{P}, \mathrm{V}$ & $4^{\circ} \mathrm{C}$ & $24 \mathrm{~h}$ & $\begin{array}{l}\text { Llenar por completo el recipiente y tapar herméticamente. } \\
\text { Evítese la agitación de la muestra y su exposición prolongada } \\
\text { al aire. Las muestras deben analizarse preferentemente in situ } \\
\text { (en particular para muestras ricas en gases disueltos). }\end{array}$ \\
\hline \multirow{2}{*}{$\begin{array}{l}\text { Nitratos } \\
\left(\mathrm{mg} / \mathrm{l} \mathrm{N}-\mathrm{NO}_{3}\right)\end{array}$} & \multirow[t]{2}{*}{$\mathrm{P}, \mathrm{V}$} & $4^{\circ} \mathrm{C}, \mathrm{H}_{2} \mathrm{SO}_{4} \mathrm{pH}<2$ & $24-48 \mathrm{~h}$ & \\
\hline & & $\begin{array}{l}\text { Filtrar } \\
\text { inmediatamente } \\
4{ }^{\circ} \mathrm{C}\end{array}$ & $24-48 h$ & \\
\hline $\begin{array}{l}\text { Nitritos } \\
\left(\mathrm{mg} / 1 \mathrm{~N}-\mathrm{NO}_{2}\right)\end{array}$ & $\mathrm{P}, \mathrm{V}$ & $4{ }^{\circ} \mathrm{C}$ & $24 \mathrm{~h}-48 \mathrm{~h}$ & \\
\hline \multirow{2}{*}{$\begin{array}{l}\text { Amonio } \\
\left(\mathrm{mg} / 1 \mathrm{NH}_{4}\right) \\
\end{array}$} & \multirow[t]{2}{*}{$\mathrm{P}, \mathrm{V}$} & $4^{\circ} \mathrm{C}, \mathrm{H}_{2} \mathrm{SO}_{4} \mathrm{pH}<2$ & $24 \mathrm{~h}$ & \\
\hline & & $4{ }^{\circ} \mathrm{C}$ & $6 \mathrm{~h}$ & \\
\hline $\begin{array}{l}\text { Ortofosfato }{ }^{1} \\
\left(\mathrm{mg} / 1 \mathrm{P}^{-} \mathrm{PO}_{4}\right)\end{array}$ & $\mathrm{VB}, \mathrm{V}$ & $\begin{array}{l}\text { Filtrar } \\
\text { inmediatament, } \\
4{ }^{\circ} \mathrm{C}\end{array}$ & $24 \mathrm{~h}$ & $\begin{array}{l}\text { Lávense todos los recipientes de vidrio con } \mathrm{HCl} \text { diluido } \\
\text { y después varias veces con agua destilada. No usar nunca } \\
\text { detergentes comerciales que contengan fosfatos para limpiar } \\
\text { el material de vidrio utilizado en este análisis. }\end{array}$ \\
\hline $\begin{array}{l}\text { Cloruros } \\
(\mathrm{mg} / \mathrm{l} \mathrm{Cl})\end{array}$ & $\mathrm{P}, \mathrm{V}$ & No especial & $1 \mathrm{mes}$ & \\
\hline $\begin{array}{l}\text { Sulfatos } \\
\left(\mathrm{mg} / 1 \mathrm{SO}_{4}\right)\end{array}$ & $\mathrm{P}, \mathrm{V}$ & $4^{\circ} \mathrm{C}$ & 1 semana & $\begin{array}{l}\text { Para aguas con alta DBO existen consideraciones } \\
\text { especiales para su conservación }\end{array}$ \\
\hline $\begin{array}{l}\text { Sólidos en } \\
\text { suspensión }(\mathrm{mg} / \mathrm{l})\end{array}$ & $\mathrm{P}, \mathrm{V}$ & No especial & $24 \mathrm{~h}$ & Las muestras deben analizarse lo antes posible \\
\hline
\end{tabular}


debe comprobar la idoneidad de este método para el análisis de cada parámetro. En este caso, el tipo de recipientes recomendados en la Tabla 3 puede verse modificado.

\section{Medida del caudal}

Se busca un transecto del río con la sección lo más homogénea posible y por la que pase todo el caudal, preferentemente con un flujo laminar para que el error cometido sea lo menor posible:

- se mide su anchura con ayuda de una cinta métrica.

- a lo ancho de este transecto se hacen medidas de profundidad (con un metro rígido) y de velocidad del agua con ayuda de un correntímetro, cada $20 \mathrm{~cm}$. o cada $50 \mathrm{~cm}$., según la anchura y homogeneidad del río. La velocidad se debe tomar a una profundidad que esté a 2/3 del fondo.

- en el caso de disponer de un correntímetro con la opción de hacer la media de la velocidad del agua en continuo, debemos subir y bajar el aparato desde la superficie hasta el fondo varias veces, de forma que obtengamos una media integrada de la velocidad del agua en cada una de los perfiles verticales en los que hemos medido la profundidad.

El valor del caudal $\left(\mathrm{m}^{3} / \mathrm{s}\right)$ en cada una de las secciones se obtiene multiplicando el área de la sección $\left(\mathrm{m}^{2}\right)$ por la velocidad media del agua $(\mathrm{m} / \mathrm{s})$ en cada una de ellas. El caudal total del transecto sería la suma de los caudales obtenidos en cada una de las secciones. 


\section{PROTOCOLO 2: IHF (ÍNDICE DE EVALUACIÓN DEL HÁBITAT FLUVIAL)}

\section{Consideraciones previas a tener en cuenta en la aplicación del índice}

\section{Pasos a seguir}

1 Seleccionar el área de observación

El tramo de río evaluado deberá tener una longitud suficiente (unos $100 \mathrm{~m}$ ) para proporcionar al observador la información necesaria que se requiere para cubrir los siete bloques de los que consta el índice.
Observaciones

El índice será aplicado durante periodos en los que el caudal sea bajo, de modo que el sustrato y las características del canal puedan verse con facilidad. No evaluar el hábitat inmediatamente después de una crecida.
2 Independencia de los bloques a analizar

Los siete bloques en los que está basado el IHF son independientes y la puntuación de cada uno de ellos no puede ser superior a la que se indica al final de la hoja de campo.
En cada bloque se valorará solamente la presencia de cada uno de los parámetros indicados, no su ausencia.

\section{Puntuación final}

La puntuación final será el resultado de la suma de los siete bloques y por lo tanto nunca puede ser superior a 100.

\section{Consideraciones útiles para rellenar la hoja de campo}

\begin{tabular}{|c|c|c|}
\hline Bloques & Consideraciones & Observaciones \\
\hline 1 & $\begin{array}{l}\text { Inclusión rápidos - sedimentación pozas } \\
\text { Inclusión: Se contabiliza el grado en que las } \\
\text { partículas del sustrato están fijadas (hundidas) en } \\
\text { el lecho del río. } \\
\text { Sedimentación: Consiste en la deposición de } \\
\text { material fino en zonas más lénticas del río. }\end{array}$ & $\begin{array}{l}\text { La inclusión se mide aguas arriba y en la parte } \\
\text { central de rápidos y zonas de piedras, donde no } \\
\text { exista una deposición de sedimentos y la } \\
\text { distribución de las partículas del sustrato pueda } \\
\text { verse con mayor claridad. }\end{array}$ \\
\hline 2 & $\begin{array}{l}\text { Frecuencia de rápidos } \\
\text { Se hace una estima promedio de la aparición de } \\
\text { rápidos con respecto a la presencia de zonas más } \\
\text { remansadas. }\end{array}$ & $\begin{array}{l}\text { En este apartado se pretende evaluar la } \\
\text { heterogeneidad del curso del río. El que se produzca } \\
\text { de forma frecuente la alternancia de rápidos con } \\
\text { pozas a la escala de tramo fluvial, asegura la } \\
\text { existencia de una mayor diversidad de hábitats para } \\
\text { la comunidad de organismos acuáticos. }\end{array}$ \\
\hline 3 & $\begin{array}{l}\text { Composición del sustrato } \\
\text { Para rellenar este apartado se hace una estima } \\
\text { visual aproximada de la composición media del } \\
\text { sustrato, siguiendo las categorías del RIVPACS } \\
\text { (River InVertebrate Prediction And Classification } \\
\text { System) (Wright et al., 1984). }\end{array}$ & $\begin{array}{l}\text { El diámetro de partícula considerado en las } \\
\text { categorías del RIVPACS es el siguiente: } \\
\text { Bloques y piedras: }>64 \mathrm{~mm} \text {. } \\
\text { Cantos y gravas: }>64 \mathrm{~mm}>2 \mathrm{~mm} \text {. } \\
\text { Arena: } 0.6-2 \mathrm{~mm} . \\
\text { Limo y arcilla: }<0.6 \mathrm{~mm} \text {. }\end{array}$ \\
\hline
\end{tabular}


Consideraciones útiles para rellenar la hoja de campo. Continuación.

\begin{tabular}{|c|c|c|}
\hline Bloques & Consideraciones & Observaciones \\
\hline 4 & $\begin{array}{l}\text { Regímenes de velocidad/ profundidad } \\
\text { La presencia de una mayor variedad de regímenes } \\
\text { de velocidad y profundidad proporciona una mayor } \\
\text { diversidad de hábitat disponibles para los } \\
\text { organismos. }\end{array}$ & $\begin{array}{l}\text { Como norma general se considera una profundidad } \\
\text { de } 0.5 \mathrm{~m} \text { para distinguir entre profundo y somero y } \\
\text { una velocidad de } 0.3 \mathrm{~m} / \mathrm{s} \text { para separar rápido de } \\
\text { lento. }\end{array}$ \\
\hline 5 & $\begin{array}{l}\text { Porcentaje de sombra en el cauce } \\
\text { Estima, de forma visual, la sombra proyectada por } \\
\text { la cubierta vegetal adyacente, que determina la } \\
\text { cantidad de luz que alcanza el canal del río e } \\
\text { influencia el desarrollo de los productores } \\
\text { primarios. }\end{array}$ & \\
\hline 6 & $\begin{array}{l}\text { Elementos heterogeneidad } \\
\text { Mide la presencia de elementos tales como hojas, } \\
\text { ramas, troncos o raíces dentro del lecho del río. } \\
\text { Estos elementos proporcionan el hábitat físico que } \\
\text { puede ser colonizado por los organismos } \\
\text { acuáticos, a la vez que constituyen una fuente de } \\
\text { alimento para los mismos. }\end{array}$ & $\begin{array}{l}\text { En este apartado se tendrá en cuenta únicamente la } \\
\text { aparición de los elementos indicados. Si no } \\
\text { existiesen no se les daría ninguna puntuación. }\end{array}$ \\
\hline 7 & $\begin{array}{l}\text { Cobertura y diversidad de vegetación acuática } \\
\text { Mide la cobertura de la vegetación acuática en el } \\
\text { cauce fluvial. La mayor diversidad de morfologías } \\
\text { en los productores primarios incrementa la } \\
\text { disponibilidad de hábitats y de fuentes de alimento } \\
\text { para muchos organismos. En la misma medida la } \\
\text { dominancia de un grupo sobre el total de la } \\
\text { cobertura no debería superar el } 50 \% \text {. }\end{array}$ & $\begin{array}{l}\text { Plocon: incluye organismos fijos al sustrato por un } \\
\text { extremo -rizoides- en muchos casos desprendidos y } \\
\text { flotando, por ejemplo, Cladophora, Zygnematales, } \\
\text { Oedogoniales y Briófitos. } \\
\text { Pecton: incluye talos aplanados, laminares o } \\
\text { esféricos, por ejemplo, Nostoc, Hildenbrandia, } \\
\text { Chaetoforales, Rivulariáceas, Fieltros de } \\
\text { oscilatorias o Perifiton de diatomeas. } \\
\text { Fanerógamas y charales: por ejemplo, especies de } \\
\text { los géneros Potamogeton, Ranunculus, } \\
\text { Ceratophyllum, Apium, Lemna, Myriophyllum, } \\
\text { Zannichellia o Rorippa y Chara } \\
\text { Briófitos: incluyen musgos y hepáticas. }\end{array}$ \\
\hline
\end{tabular}




\section{PROTOCOLO 3: IBMWP "IBERIAN BIOLOGICAL MONITORING WORKING PARTY"}

\section{Consideraciones previas a tener en cuenta en la aplicación del índice:}

\section{Pasos a seguir}

\section{Seleccionar el área de observación}

El tramo de río evaluado deberá tener una longitud aproximada de $100 \mathrm{~m}$. Se realizará un recorrido visual a lo largo del tramo a muestrear y se identificarán los diferentes hábitats para macroinvertebrados presentes: zonas lóticas o leníticas, con macrófitos o no, con raíces o con diferentes tipos de sustratos: arena, limo, etc.

\section{Observaciones}

El índice no se deberá aplicar inmediatamente después de una crecida, ni inmediatamente después de un periodo en que el cauce haya estado seco. En ambos casos debe esperarse al menos un mes antes de realizar el muestreo

\section{Muestreo de los hábitats}

Una vez recorrida la zona y localizados los diferentes microhábitats, antes de introducirse en el agua es importante localizar animales esquivos que viven en la superficie como Gyrinidae, Gerridae o Hydrometridae, ya que tratan de huir rápidamente y podrían pasar desapercibidos si se lleva a cabo el muestreo de inmediato. A continuación se muestrearán todos los hábitats presentes con una red de mano de $300 \mu \mathrm{m} *$ de luz de malla y una boca de entrada de unos $30 \mathrm{~cm}$ de diámetro. El muestreo se realizará colocando la malla a contracorriente y removiendo el sustrato aguas arriba de la manga con la mano o el pie, realizando un movimiento zigzagueante con la red para que todo el material removido entre a través de ésta.

Las piedras deben limpiarse bien dentro de la red o en una batea por ambas caras, así como troncos, raíces, masas de algas, etc.
El muestreo se realiza desde aguas abajo hacia aguas arriba del tramo para evitar que la perturbación haga huir a los animales.

Para evitar que al colmatarse la red la corriente ayude a los animales a escapar se debe vaciar a menudo el contenido de ésta en bateas de plástico blanco.

3 Identificación de los taxones

Se proponen dos protocolos de identificación dependiendo de si se está muestreando una estación de referencia o no. En caso del muestreo de una estación de referencia se realiza un mayor esfuerzo en la identificación de los taxones, ya que se prevé que estas localidades serán muchos más diversas que las de "no referencia". Además, en estas localidades, se propone la toma de datos de abundancia relativa en laboratorio, para un posible posterior tratamiento de los datos. En caso de que no interese tal tratamiento será suficiente con aplicar el Protocolo I a todas las localidades de muestreo.

3.1 "Estaciones de no referencia". Protocolo I.

En el campo se toma una batea blanca de plástico y se llena de agua. El contenido de las redadas se deposita en la batea asegurándose de que no queda ningún individuo adherido a la red. Se capturan los diferentes taxones con ayuda de unas pinzas finas o un aspirador entomológico y se van identificando a medida que se localizan en la batea. Los taxones que son dudas y que quedan sin identificar se introducen en un vial con alcohol de $70^{\circ}$. Y el material revisado de la batea es devuelto al río.
El muestreo debe continuar hasta que nuevas redadas no aporten nuevos taxones. La duración de esta operación depende de la experiencia y habilidad del operador. Entre distintos puntos de muestreo se han de lavar bien las bateas y las redes para evitar llevar individuos de unos puntos de muestreo a otros.

* Si la corriente es muy fuerte, utilizar tamaños de malla de $500 \mu \mathrm{m}$. 
Consideraciones previas a tener en cuenta en la aplicación del índice. Continuación.

Pasos a seguir

\section{2. "Estaciones de referencia". Protocolo II.}

Se procede igual que en el caso anterior pero de cada taxón nos aseguramos capturar al menos de 1-3 individuos, que se introducen en un vial con alcohol de $70^{\circ}$. Todo el material sobrante después de las redadas se introduce en un bote de boca ancha o en una bolsa hermética de plástico y se fija con Formol al $4 \%$. En el laboratorio se submuestrea separando 200 individuos al azar (Bonada et al., en este volumen) y se revisa la muestra por si algún taxón escaso o críptico no se hubiera detectado en el submuestreo y además hubiese escapado al muestreo de campo. De esta forma se puede obtener sumando los animales separados en campo y los separados en laboratorios un dato semicuantitativo que nos permita calcular las abundancias relativas de los distintos taxones.
Observaciones

Dada la elevada toxicidad del formol, la muestra se puede conservar igualmente en alcohol de $70^{\circ}$. En este último caso el volumen de fijador en el bote ha de ser mucho mayor y la muestra no se podrá conservar por mucho tiempo en el laboratorio.

\section{Cálculo del índice}

Para el cálculo del índice se suman las puntuaciones parciales que se obtienen de la presencia de cada familia y de esta forma se obtiene la puntuación global del punto de muestreo. Si en el tramo aparecen más de un individuo de una familia esta sólo puntuará una vez.

Los rangos de calidad según el índice IBMWP son:

\begin{tabular}{|c|c|c|c|}
\hline Estado Ecológico & CALIDAD & IBMWP & Color \\
\hline Muy Bueno & Buena. Aguas no contaminadas o no alteradas de modo sensible. & $\geq 101$ & Azul \\
\hline Bueno & Aceptable. Son evidentes algunos efectos de contaminación. & $61-100$ & Verde \\
\hline Aceptable $(=$ Moderado $) *$ & Dudosa. Aguas contaminadas. & $36-60$ & Amarillo \\
\hline Deficiente & Crítica. Aguas muy contaminadas. & $16-35$ & Naranja \\
\hline Malo & Muy critica. Aguas fuertemente contaminadas & $<15$ & Rojo \\
\hline
\end{tabular}

* Hay que señalar que en la versión inglesa de la Directiva Marco del agua, se lee "Moderate", que ha sido traducida al castellano como "Aceptable" en vez de como "Moderado", indicando que existe una alteración (ver Ortiz, en este volumen)

El problema de establecer límites estrictos de calidad de aguas implica la necesidad de reconocer situaciones intermedias entre unos y otros. Es por ello que aquellos valores que queden cinco unidades por exceso o por defecto de los límites establecidos en la tabla anterior han de considerarse entre dos clases de calidad, alternando los colores representativos de las clases de calidad correspondientes (Alba-Tercedor, 1996). Por ejemplo, 103 quedaría intermedio entre las clases I y II y debería colorearse en la cartografía con azul y verde. Estos rangos de calidad están siendo revisados en la segunda fase del proyecto GUADALMED. 


\section{PROTOCOLO 4: QBR (ÍNDICE DE CALIDAD DEL BOSQUE DE RIBERA)}

\section{Consideraciones previas a tener en cuenta en la aplicación del índice:}

\section{Pasos a seguir}

\section{Seleccionar el área de observación}

Se debe escoger un tramo de $100 \mathrm{~m}$ de longitud, aguas arriba del punto de muestreo, y se considera toda la anchura potencial del bosque de ribera para calcular el QBR. Se delimitará visualmente la orilla y la ribera (ver dibujo de la hoja de campo de este índice) de la zona riparia.

En las estaciones de referencia se considerará el tramo de río que tome los mayores valores de QBR.

\section{Observaciones}

Orilla. Zona del cauce inundable en crecidas periódicas en un período aproximado de dos años.

Ribera. Zona inundable en crecidas de gran magnitud (períodos de hasta 100 años). Pueden estar incluidas varias terrazas aluviales.

El índice no es aplicable en las zonas altas de montaña, donde no existe de manera natural vegetación arbórea.

2 Independencia de los bloques a analizar

Los cuatro bloques en los que está basado el QBR son totalmente independientes y la puntuación de cada uno de ellos no puede ser negativa ni superior a 25 .

\section{Cálculo bloque por bloque}

En cada bloque se escoge una de las cuatro opciones principales, puntuando $25,10,5$ ó 0 . Solamente se puede escoger una entrada: La que cumpla la condición exigida siempre leyendo de arriba abajo.

La puntuación final de cada bloque será modificada por las condiciones expuestas en la parte inferior de cada bloque, tantas veces como se cumpla la condición (sumando o restando).
De las cuatro opciones principales, se escogerá solamente una de ellas.

La puntuación final de cada bloque tendrá un 25 como máximo y un 0 como mínimo.

Las condiciones se analizarán considerando ambos

márgenes del río como una única unidad.

\section{Puntuación final}

La puntuación final será el resultado de la suma de los cuatro bloques, por tanto variará entre 0 y 100 .

\section{Nota}

Los puentes y caminos utilizados para acceder a la estación de muestreo no se tendrán en cuenta para la evaluación del índice QBR. Si es posible, el QBR debería ser analizado aguas arriba y debajo de estos accesos. Otros puentes o carreteras (por ejemplo las paralelas al río) sí que deberán ser consideradas.
Los tramos de ribera cercanos a la zona de acceso al río suelen estar perturbados y pueden hacer disminuir la puntuación.

Si es posible, es interesante realizar varios transectos (cada 100-200 m) y evaluar el QBR en un tramo largo para tener una puntuación más representativa de la zona. 


\section{Consideraciones útiles para rellenar la hoja de campo:}

\begin{tabular}{|c|c|c|}
\hline Bloques & Consideraciones & Observaciones \\
\hline 1 & $\begin{array}{l}\text { Grado de cobertura riparia } \\
\text { Se contabiliza el porcentaje de cobertura de toda } \\
\text { la vegetación, exceptuando las plantas de } \\
\text { crecimiento anual. Se consideran ambos lados del } \\
\text { río de forma conjunta. } \\
\text { Hay que tener en cuenta también la conectividad } \\
\text { entre el bosque de ribera y el ecosistema forestal } \\
\text { adyacente para sumar o restar puntos. }\end{array}$ & $\begin{array}{l}\text { Nos interesa puntuar la cobertura del terreno por la } \\
\text { vegetación, sin tener en cuenta su estructura vertical, } \\
\text { que se evalúa en el siguiente apartado. En este } \\
\text { bloque se destaca el papel de la vegetación como } \\
\text { elemento estructurador del ecosistema de ribera. } \\
\text { Los caminos sin asfalto de menos de } 4 \text { metros de } \\
\text { ancho no se consideran como elementos de } \\
\text { aislamiento con el ecosistema adyacente. }\end{array}$ \\
\hline
\end{tabular}

\section{Estructura de la cobertura}

La puntuación se realiza según el porcentaje de cobertura de árboles y, en ausencia de éstos, arbustos sobre la totalidad de la zona a estudiar. Se considera riberas a ambos márgenes del río. Elementos como la linealidad en los pies de los árboles (síntomas de plantaciones), o las coberturas distribuidas no uniformemente y formando manchas se penalizan en el índice, mientras que la presencia de helófitos en la orilla y la interconexión entre árboles y arbustos en la ribera, se potencian.
En este apartado lo que se pretende evaluar es la complejidad de la vegetación que puede ser causa de una mayor biodiversidad animal y vegetal en la zona. Cuando debido a las características geomorfológicas o hidrológicas del tramo, el bosque adyacente ocupa la zona riparia, éste se contabiliza en el apartado de cobertura y estructura (bloque 1 y 2), pero no para evaluar la calidad de la cobertura (bloque 3 ).

\section{Calidad de la cobertura}

Para rellenar este apartado, antes que nada hay que determinar el tipo geomorfológico con las indicaciones del reverso de la hoja de campo. Después de haber seleccionado el tipo geomorfológico (1 a 3 ) contaremos el número de especies arbóreas o arbustivas nativas riparias. Los bosques en forma de túnel a lo largo del río suponen un aumento de la puntuación, dependiendo del porcentaje de cobertura a lo largo del tramo estudiado.

La disposición de las diferentes especies arbóreas en galería, desde la zona más cercana al río hasta el final de la zona de ribera, puntúan aumentando el valor del índice.
Para determinar el tipo geomorfológico hay que utilizar el reverso de la hoja de campo. En esta parte puntuaremos el margen izquierdo y derecho en función de su desnivel y forma. La puntuación final se obtiene sumando los valores de ambos márgenes y complementando este valor con las restas y las sumas de los apartados inferiores (si es necesario). La presencia de islas en el río decrecen la puntuación, mientras que la presencia de un suelo rocoso y duro (lascas) con baja potencialidad para enraizar una buena vegetación de ribera, la aumentan. El resultado de la operación nos indica el tipo geomorfológico del canal del tramo a estudiar y lo usaremos para seguir por una u otra columna en el tercer bloque.

Las especies introducidas en la zona y naturalizadas penalizan en esta parte del índice. Existe una lista de las especies introducidas (consideradas no naturales) más frecuentes en los ríos muestreados en el reverso de la hoja de campo.

\section{$4 \quad$ Naturalidad del canal fluvial}

La modificación de las terrazas adyacentes al río supone la reducción del cauce, el aumento de la pendiente de los márgenes y la pérdida de sinuosidad en el río. Los campos de cultivo cercanos al río y las actividades extractivas producen este efecto.

Cuando existan estructuras sólidas, como paredes, muros, etc., los signos de alteración son más evidentes y la puntuación baja.
No se consideran los puentes ni los pasos para cruzar el río que nos permiten acceder a la estación de muestreo. 
Los rangos de calidad* según el índice QBR son:

\begin{tabular}{lll}
\hline Nivel de calidad & QBR & Color representativo \\
\hline Bosque de ribera sin alteraciones, calidad muy buena, estado natural & $\geq 95$ & Azul \\
Bosque ligeramente perturbado, calidad buena & $75-90$ & Verde \\
Inicio de alteración importante, calidad intermedia & $55-70$ & Amarillo \\
Alteración fuerte, mala calidad & $30-50$ & Naranja \\
Degradación extrema, calidad pésima & $\leq 25$ & Rojo \\
\hline
\end{tabular}

* Los rangos de calidad son provisionales, están sujetos a revisión en la segunda fase del proyecto GUADALMED.

\section{BIBLIOGRAFÍA}

ALBA-TERCEDOR, J. \& A. SÁNCHEZ-ORTEGA. 1988. Un método rápido y simple para evaluar la calidad biológica de las aguas corrientes basado en el de Hellawell (1978). Limnetica, 4: 51-56.

ALBA-TERCEDOR, J. 1996. Macroinvertebrados acuáticos y calidad de las aguas de los ríos. $I V$ Simposio del Agua en Andalucía (SIAGA), Almería, España: 203-213.

ALBA-TERCEDOR, J. \& A. PUJANTE. 2000. Running-water biomonitoring in Spain. Opportunities for a predictive approach. In: Assessing the Biological Quality of Freshwater: RIVPACS and similar techniques. J.F. Wright, D.W. Sutcliffe \& M. Furse (eds.): 207-216. Freshwater Biological Association.

ALBA-TERCEDOR, J., P. JÁIMEZ-CUÉLLAR, M. ÁLVAREZ, J. AVILÉS, N. BONADA, J. CASAS, A. MELLADO, M. ORTEGA, I. PARDO, N. PRAT, M. RIERADEVALL, S. ROBLES, C. E. SÁINZ-CANTERO, A. SÁNCHEZ-ORTEGA, M. L. SUÁREZ, M. TORO, M. R. VIDALABARCA, S. VIVAS \& C. ZAMORA-MUÑOZ. 2002. Caracterización del estado ecológico de ríos mediterráneos ibéricos mediante el índice IBMWP (=BMWP'). Limnetica, 21: 175-185.

AENOR. 1997. Recopilación de Normas UNE. Medio Ambiente. Tomo 1: Calidad del Agua. Madrid: Asociación Española de Normalización y Certificación.

APHA; AWWA; WPCF. 1992. Standar Methods for the examination of water and waste water, $17^{\text {th }} \mathrm{ed}$. American Public Health Association.

BARBOUR, M. T., J. GERRITSEN, B. D. SNYDER \& J. B. STRIBLING. 1999. Rapid Bioassessment Protocols for Use in Streams and Wadeable Rivers: Periphyton, Benthic Macroinvertebrates and Fish, $2^{\text {ond }}$ ed. EPA 841-B-99-002. US EPA, Office of Water, Washington D.C., USA.

BONADA, N., N. PRAT, A. MUNNÉ, M. PLANS, C. SOLÀ, M. ÁlVAREZ, I. PARDO, G. MOYÀ, G. RAMON, M. TORO, S. ROBLES, J. AVILÉS, M.L. SUÁREZ, M. R. VIDAL-ABARCA, A. MELLADO, J. L. MORENO, C. GUERRERO, S. VIVAS, M. ORTEGA, J. CASAS, A. SÁNCHEZ-ORTEGA, P. JÁIMEZ-CUÉLLAR \& J. ALBA-TERCEDOR. 2002. Intercalibración de la metodología GUADALMED. Selección de un protocolo de muestreo para la determinación del estado ecológico de los ríos mediterráneos. Limnetica, 21: 13-33.

DAVIES, P.E. 1994. National River Processes and Management Program Monitoring River Health Initiative. River Bioassessment Manual Version 1.0. Department of the Environment, Sport and Territories, Canberra.

D.O.C.E. 2000. Directiva 2000/60/CE del Parlamento Europeo y del Consejo de 23 de octubre de 2000 por la que se establece un marco comunitario de actuación en el ámbito de la política de aguas. D.O.C.E. L 327 de 22.12.00. 69 pp.

MUNNÉ, A., C. SOLÀ \& N. PRAT. 1998. QBR: Un índice rápido para la evaluación de la calidad de los ecosistemas de ribera. Tecnología del Agua, 175: 20-37.

MUNNÉ, A., C. SOLÀ, M. RIERADEVALL \& N. PRAT. 1998. Índex QBR. Mètode per a l'avaluació de la qualitat dels ecosistemes de ribera. Barcelona: Diputació de Barcelona, Àrea de Medi Ambient.

MUNNÉ, A., N. PRAT, C. SOLÀ, N. BONADA \& M. RIERADEVALL. 2003. A simple field method for assessing the ecological quality of riparian habitat in rivers and streams. QBR index. Aquatic Conserv.: Mar. Freshw. Ecosyst., 13: 147-164. 
ORTIZ, J.L. 2002. La Directiva Marco del Agua: Aspectos relevantes para el proyecto GUADALMED. Limnetica, 21: 5-12.

PARDO, I., M. ÁlVAREZ, J. CASAS, J. L. MORENO, S. VIVAS, N. BONADA, J. ALBATERCEDOR, P. JÁIMEZ-CUÉLLAR, G. MOYÀ, N. PRAT, S. ROBLES, M. L. SUÁREZ, M. TORO \& M. R. VIDAL-ABARCA. 2002. El hábitat de los ríos mediterráneos. Diseño de un índice de diversidad de hàbitat. Limnetica, 21: 115-132.

PLAFKIN, J. L., M. T. BARBOUR, K. D. PORTER, S. K. GROSS \& R. M. HUGHES. 1989. Rapid Bioassessment protocols for use in streams and rivers: benthic macroinvertebrates and fish. EPA/444/4-89-001. United Estates Environmental Protection Agency, Washington D. C., USA.

PRAT, N., A. MUNNÉ, M. RIERADEVALL, C. SOLÀ \& N. BONADA. 2000. ECOSTRIMED. Protocolo para determinar el estado ecológico de los ríos mediterráneos. Barcelona: Diputació de Barcelona, Àrea de Medi Ambient.
RESH, V. H., R. H. NORRIS \& M. T. BARBOUR. 1995. Design and implementation of rapid assessment approaches for water resource monitoring using benthic macroinvertebrates. Aust. J. Ecol., 20: 108-121.

RESH, V. H. \& J. K. JACKSON. 1993. Rapid assessment approaches to biomonitoring using benthic macroinvertebrates. In: Freshwater Biomonitoring and Benthic Macroinvertebrates. D. M. ROSENBERG \& V. H. RESH (eds.): 195-233. Chapman and Hall, New York, USA.

SUÁREZ, M. L. \& M. R. VIDAL-ABARCA. 2000. Aplicación del índice de calidad del bosque de ribera, QBR (Munné et al., 1998) a los cauces fluviales de la cuenca del río Segura. Tecnología del Agua, 201: 33-45.

WRIGTH, J. F., D. MOSS, P. D. ARMITAGE. \& M. T. FURSE. 1984. A preliminary classification of running water sites in Great Britain based on macroinvertebrate species and the prediction of community type using environmental data. Freshwat. Biol., 14:221-256. 
Anexo. Hojas de campo del IBMWP, IHF y QBR usadas en el proyecto GUADALMED. Field sheets for the IBMWP, IHF and QBR indices used in the Guadalmed project.

\section{IBMWP}

Río:

Fecha:

Localidad:

Identificado por:

\begin{tabular}{|c|c|c|c|c|c|c|c|c|}
\hline TAXÓN & PTS & Abund & TAXÓN & PTS & Abund & TAXÓN & PTS & Abund \\
\hline TRICLADIDA & & & ODONATA & & & TRICHOPTERA & & \\
\hline Dendrocoelidae & 5 & & Aeshnidae & 8 & & Beraeidae & 10 & \\
\hline Dugesiidae & 5 & & Calopterygidae & 8 & & Brachycentridae & 10 & \\
\hline Planariidae & 5 & & Coenagrionidae & 6 & & Calamoceratidae & 10 & \\
\hline OLIGOCHAETA & 1 & & Cordulegasteridae & 8 & & Ecnomidae & 7 & \\
\hline HIRUDINEA & & & Corduliidae & 8 & & Glossosomatidae & 8 & \\
\hline Erpobdellidae & 3 & & Gomphidae & 8 & & Goeridae & 10 & \\
\hline Glossiphoniidae & 3 & & Lestidae & 8 & & Hydropsychidae & 5 & \\
\hline Hirudidae & 3 & & Libellulidae & 8 & & Hydroptilidae & 6 & \\
\hline Piscicolidae & 4 & & Platycnemididae & 6 & & Lepidostomatidae & 10 & \\
\hline MOLLUSCA & & & PLECOPTERA & & & Leptoceridae & 10 & \\
\hline Ancylidae & 6 & & Capniidae & 10 & & Limnephilidae & 7 & \\
\hline Bithyniidae & 3 & & Chloroperlidae & 10 & & Molannidae & 10 & \\
\hline Ferrissidae & 6 & & Leuctridae & 10 & & Odontoceridae & 10 & \\
\hline Hydrobiidae & 3 & & Nemouridae & 7 & & Philopotamidae & 8 & \\
\hline Lymnaeidae & 3 & & Perlidae & 10 & & Phryganeidae & 10 & \\
\hline Neritidae & 6 & & Perlodidae & 10 & & Polycentropodidae & 10 & \\
\hline Physidae & 3 & & Taeniopterygidae & 10 & & Psychomyiidae & 8 & \\
\hline Planorbidae & 3 & & HETEROPTERA & & & Rhyacophilidae & 7 & \\
\hline Sphaeriidae & 3 & & Aphelocheiridae & 10 & & Sericostomatidae & 10 & \\
\hline Thiaridae & 6 & & Corixidae & 3 & & Thremmatidae & 10 & \\
\hline Unionidae & 6 & & Gerridae & 3 & & LEPIDOPTERA & & \\
\hline Valvatidae & 3 & & Hydrometridae & 3 & & Pyralidae & 4 & \\
\hline Viviparidae & 6 & & Mesoveliidae & 3 & & DIPTERA & & \\
\hline HYDRACARINA & 4 & & Naucoridae & 3 & & Athericidae & 10 & \\
\hline OSTRACODA & 3 & & Nepidae & 3 & & Blephariceridae & 10 & \\
\hline AMPHIPODA & & & Notonectidae & 3 & & Ceratopogonidae & 4 & \\
\hline Corophiidae & 6 & & Pleidae & 3 & & Chironomidae & 2 & \\
\hline Gammaridae & 6 & & Veliidae & 3 & & Culicidae & 2 & \\
\hline ISOPODA & & & NEUROPTERA & & & Dixidae & 4 & \\
\hline Asellidae & 3 & & Sialidae & 4 & & Dolichopodidae & 4 & \\
\hline DECAPODA & & & COLEOPTERA & & & Empididae & 4 & \\
\hline Astacidae & 8 & & Chrysomelidae & 4 & & Ephydridae & 2 & \\
\hline Atyidae & 6 & & Curculionidae & 4 & & Limoniidae & 4 & \\
\hline Palaemonidae & 6 & & Dryopidae & 5 & & Muscidae & 4 & \\
\hline EPHEMEROPTERA & & & Dytiscidae & 3 & & Psychodidae & 4 & \\
\hline Baetidae & 4 & & Elmidae & 5 & & Ptychopteridae & 4 & \\
\hline Caenidae & 4 & & Gyrinidae & 3 & & Rhagionidae & 4 & \\
\hline Ephemerellidae & 7 & & Haliplidae & 4 & & Sciomyzidae & 4 & \\
\hline Ephemeridae & 10 & & Helodidae & 3 & & Simuliidae & 5 & \\
\hline Heptageniidae & 10 & & Hydraenidae & 5 & & Stratiomyidae & 4 & \\
\hline Leptophlebiidae & 10 & & Hydrochidae & 5 & & Syrphidae & 1 & \\
\hline Oligoneuriidae & 5 & & Hydrophilidae & 3 & & Tabanidae & 4 & \\
\hline Polymitarcidae & 5 & & Hygrobiidae & 3 & & Thaumaleidae & 2 & \\
\hline Potamanthidae & 10 & & Noteridae & 3 & & Tipulidae & 5 & \\
\hline Prosopistomatidae & 7 & & Psephenidae & 3 & & & & \\
\hline Siphlonuridae & 10 & & Scirtidae & 3 & & & & \\
\hline
\end{tabular}

Número ind.

Abund.

Otros organismos

\begin{tabular}{cc}
\hline $1-3$ & 1 \\
$4-10$ & 2 \\
$11-100$ & 3 \\
$>100$ & 4 \\
\hline
\end{tabular}

Cambaridae

Dreissenidae

Hydridae 


\section{Evaluación del Hábitat Fluvial para Ríos Mediterráneos. Índice IHF}

\begin{tabular}{|l|l|}
\hline Estación \\
\hline Fecha \\
\hline Operador \\
\hline
\end{tabular}

Bloques

1. Inclusión rápidos-sedimentación pozas

\begin{tabular}{l|l}
\hline Rápidos & Piedras, cantos y gravas no fijadas por sedimentos finos. Inclusión $0-30^{\circ}$
\end{tabular}

Piedras, cantos y gravas poco fijadas por sedimentos finos. Inclusión $30-60 \%$

Piedras, cantos y gravas medianamente fijadas por sedimentos finos. Inclusión $>60 \%$

Sólo pozas $\quad$ Sedimentación $0-30 \%$

Sedimentación $30-60 \%$

Sedimentación $>60 \%$

Puntur

2. Frecuencia de rápidos
\[ \text { Alta frecuencia de rápidos. Relación distancia entre rápidos / anchura del río < } 7 \]

\begin{tabular}{|l|}
\hline Alta frecuencia de rápidos. Relación distancia entre rápidos / anchura del río $<7$ \\
\hline Escasa frecuencia de rápidos. Relación distancia entre rápidos / anchura del rio 7 - 15 \\
\hline Ocura
\end{tabular}

Ocurrencia ocasional de rápidos. Relación distancia entre rápidos / anchura del río $15-25$

Constancia de flujo laminar o rápidos someros. Relación distancia entre rápidos/anchura del río $>25$

Sólo pozas

3. Composición del substrato

\begin{tabular}{|l|l|}
\hline \multirow{2}{*}{$\%$ Bloques y piedras } & $1-10 \%$ \\
\cline { 2 - 2 } & $>10 \%$ \\
\hline \multirow{2}{*}{$\%$ Cantos y gravas } & $1-10 \%$ \\
\cline { 2 - 2 }$\%$ Arena & $>10 \%$ \\
\hline \multirow{2}{*}{$\%$ Limo y arcilla } & $1-10 \%$ \\
\cline { 2 - 2 } & $>10 \%$ \\
\cline { 2 - 2 } & $1-10 \%$ \\
\hline
\end{tabular}

TOTAL (una categoria)

TOTAL (una categoria)

\begin{tabular}{|c|c|l|}
\hline & 10 & \\
\hline & 5 & \\
\hline & 0 & \\
\hline & 10 & \\
\hline & 5 & \\
\hline & 0 & \\
\hline \multicolumn{2}{|c|}{} \\
\cline { 2 - 3 }
\end{tabular}

$\mid$\begin{tabular}{l|l}
-10 & -10 \\
\hline & -10
\end{tabular}

\begin{tabular}{|r|}
\hline \\
\hline \\
\hline
\end{tabular}

\section{Regímenes de velocidad / profundidad}

\begin{tabular}{|l|l|l|}
\hline $10 \%$ & 2 & \\
\hline $0 \%$ & 5 & \\
\hline $10 \%$ & 2 & \\
\hline $0 \%$ & 5 & \\
\hline $10 \%$ & 2 & \\
\hline $0 \%$ & 5 & \\
\hline $10 \%$ & 2 & \\
\hline $10 \%$ & 5 & \\
\hline \multicolumn{2}{r|}{ TOTAL (sumar categorías) } & \\
\cline { 2 - 4 } & &
\end{tabular}

somero: $<0.5 \mathrm{~m}$

lento: $<0.3 \mathrm{~m} / \mathrm{s}$

4 categorias. Lento-profundo, lento-somero, rápido-profundo y rápido-somero

Sólo 3 de las 4 categorias

Sólo 2 de las 4

Sólo 1 de las cuatro

5. Porcentaje de sombra en el cauce

\begin{tabular}{|c|c|}
\hline Sombreado con ventanas & 10 \\
\hline Totalmente en sombra & 7 \\
\hline Grandes claros & 5 \\
\hline Expuesto & 3 \\
\hline
\end{tabular}

6. Elementos heterogeneidad

\begin{tabular}{|l}
\hline Hojarasca \\
\hline Presencia de troncos y ramas \\
\hline Raíces expuestas \\
\hline Diques naturales \\
\hline
\end{tabular}

$>10 \%$ ó $<75 \%$

$<10 \%$ o $>75 \%$

TOTAL (sumar categorías)

7. Cobertura de vegetación acuática

\begin{tabular}{|l|l|c|c|}
\hline \multirow{2}{*}{$\%$ Plocon + briófitos } & $10-50 \%$ & 10 & \\
\cline { 2 - 5 } & $<10 \% \dot{0}>50 \%$ & 5 & \\
\hline \multirow{2}{*}{$\%$ Pecton } & $10-50 \%$ & 10 & \\
\cline { 2 - 5 } & $<10 \% \dot{0}>50 \%$ & 5 & \\
\hline \multirow{2}{*}{$\%$ Fanerógamas + Charales } & $10-50 \%$ & 5 & \\
\cline { 2 - 4 } & $<10 \%>50 \%$ & TOTAL (sumar categorías) & \\
\hline
\end{tabular}

PUNTUACIÓN FINAL (suma de las puntuaciones anteriores)

La puntuación de cada uno de los apartados no puede exceder la expresada en la siguiente tabla

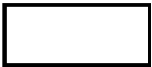

\begin{tabular}{|l|c|}
\hline Inclusión rápidos - sedimentación pozas & 10 \\
\hline Frecuencia de rápidos & 10 \\
\hline Composición del substrato & 20 \\
\hline Régimen velocidad / profundidad & 10 \\
\hline Porcentaje de sombra en el cauce & 10 \\
\hline Elementos de heterogeneidad & 10 \\
\hline Cobertura de vegetación acuática & 30 \\
\hline
\end{tabular}




\section{CALIFICACIÓN DE LA ZONA DE RIBERA DE LOS ECOSISTEMAS FLUVIALES. ÍNDICE QBR}

Esta calificación debe ser aplicada en toda la zona de ribera de los rios (orilla y ribera propiamente dicha).

Zonas inundadas periódicamente por las avenidas ordinarias y las máximas.

Los cálculos se realizarán sobre el área que presenta una potencialidad de soportar una masa vegetal en la ribera. No se contemplan las zonas con sustrato duro donde no puede enraizar una masa vegetal permanente.

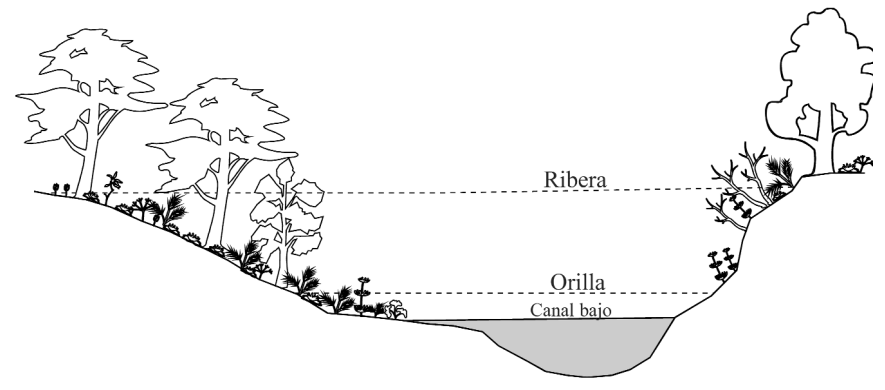

La puntuación de cada uno de los 4 apartados no puede ser negativa ni exceder de 25

Estación

Fecha

\section{Grado de cubierta de la zona de ribera}

$\begin{array}{cl}\begin{array}{c}\text { Puntuación } \\ \mathbf{2 5}\end{array} & >80 \% \text { de cubierta vegetal de la zona de ribera (las plantes anuales no se contabilizan) } \\ \mathbf{1 0} & 50-80 \% \text { de cubierta vegetal de la zona de ribera } \\ \mathbf{5} & 10-50 \% \text { de cubierta vegetal de la zona de ribera } \\ \mathbf{0} & <10 \% \text { de cubierta vegetal de la zona de ribera } \\ +10 & \text { si la conectividad entre el bosque de ribera y el ecosistema forestal adyacente es total } \\ +5 & \text { si la conectividad entre el bosque de ribera y el ecosistema forestal adyacente es superior al } 50 \% \\ -5 & \text { si la conectividad entre el bosque de ribera y el ecosistema forestal adyacente es entre el } 25 \text { y } 50 \% \\ -10 & \text { si la conectividad entre el bosque de ribera y el ecosistema forestal adyacente es inferior al } 25 \%\end{array}$

Estructura de la cubierta (se contabiliza toda la zona de ribera)

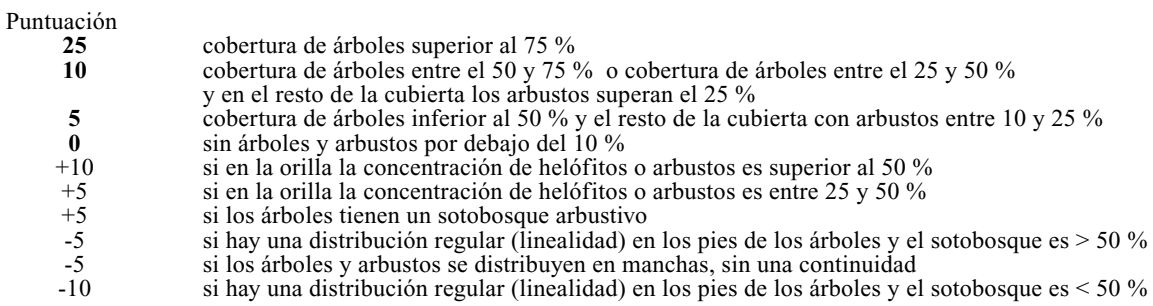

\begin{tabular}{|c|c|c|c|c|}
\hline \multicolumn{3}{|c|}{ Calidad de la cubierta (depende del tipo geomorfológico de la zona de ribera*) } & \multicolumn{2}{|c|}{ Puntuación entre 0 y 2} \\
\hline Puntuación & & Tipo 1 & Tipo 2 & Tipo 3 \\
\hline 25 & número de especies de árboles o arbustos autóctonos & $>1$ & $>2$ & $>3$ \\
\hline 10 & número de especies de árboles o arbustos autóctonos & 1 & 2 & 3 \\
\hline 5 & número de especies de árboles o arbustos autóctonos & - & 1 & $1-2$ \\
\hline 0 & sin árboles autóctonos & & & \\
\hline+10 & $\begin{array}{l}\text { si la comunidad forma una franja longitudinal continua adyacente al canal fluvial } \\
\text { en más del } 75 \% \text { de la longitud del tramo }\end{array}$ & & & \\
\hline+5 & $\begin{array}{l}\text { si la comunidad forma una franja longitudinal continua adyacente al canal fluvial } \\
\text { entre el } 50 \text { y } 75 \% \text { de la longitud del tramo }\end{array}$ & & & \\
\hline+5 & si las distintas especies se disponen en bandas paralelas al río & & & \\
\hline+5 & si el número de especies de arbustos es: & $>2$ & $>3$ & $>4$ \\
\hline-5 & si hay estructuras construidas por el hombre & & & \\
\hline-5 & si hay alguna sp. de árbol y/o arbusto alóctono** aislada & & & \\
\hline-10 & si hay sp. de árboles y/o arbustos alóctonos** formando comunidades & & & \\
\hline-10 & si hay vertidos de basuras & & & \\
\hline
\end{tabular}

Puntuación

25 el canal del río no ha estado modificado

10 modificaciones de las terrazas adyacentes al lecho del río con reducción del canal

$\mathbf{5}$ signos de alteración y estructuras rígidas intermitentes que modifican el canal del río

0 río canalizado en la totalidad del tramo

-10 si existe alguna estructura sólida dentro del lecho del río

-10 si existe alguna presa $<0>$ U otra infraestructura transversal en el lecho del río

Puntuación final (suma de las anteriores puntuaciones)

* Determinación del tipo geomorfológico de la zona de ribera (apartado 3, calidad de la cubierta)

Sumar el tipo de desnivel de la derecha y la izquierda de la orilla, y sumar o restar según los otros dos apartados. 
Puntuación

Tipos de desnivel de la zona ripària

Izquierda Derecha

Vertical/cóncavo (pendiente $>75^{\circ}$ ), con una altura no superable por las máximas avenidas
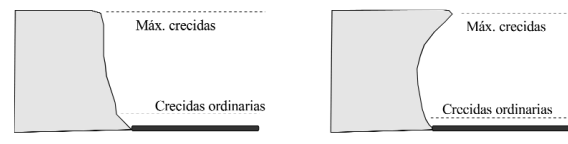

6

Igual pero con un pequeño talud o orilla inundable periódicamente (avenidas ordinarias)
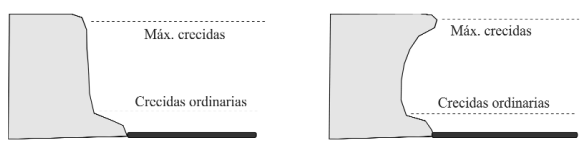

5

Pendiente entre el 45 y $75^{\circ}$, escalado o no. La pendiente se cuenta con el ángulo entre la horizontal y la recta entre la orilla y el último punto de la ribera. $\Sigma \mathrm{a}>\Sigma \mathrm{b}$

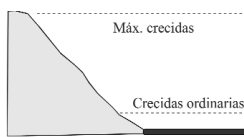

3

Pendiente entre el 20 y $45^{\circ}$, escalonado o no. $\Sigma \mathrm{a}<\Sigma \mathrm{b}$
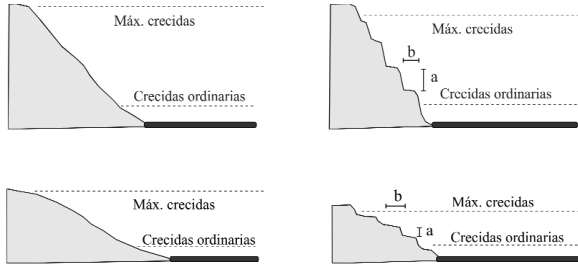

Pendiente $<20^{\circ}$, ribera uniforme y llana.

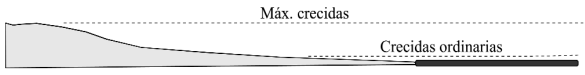

1

1

\section{Existencia de una isla o islas en el medio del lecho del río}

Anchura conjunta "a" $>5 \mathrm{~m}$.
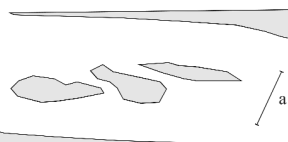

Anchura conjunta "a" entre 1 y $5 \mathrm{~m}$.

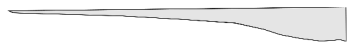

Porcentaje de sustrato duro con incapacidad para enraizar una masa vegetal permanente

$>80 \%$

$60-80 \%$

$30-60 \%$

$20-30 \%$

No se puede medir

$+6$

$+4$

$+2$

Puntuación total

Tipo geomorfológico según la puntuación

$>8$ Tipo 1 Riberas cerradas, normalmente de cabecera, con baja potencialidad de un extenso bosque de ribera entre 5 y 8 Tipo 2 Riberas con una potencialidad intermedia para soportar una zona vegetada, tramos medios de los ríos

$<5$ Tipo 3 Riberas extensas, tramos bajos de los ríos, con elevada potencialidad para poseer un bosque extenso

\section{Especies frecuentes y consideradas alóctonas}

$\begin{array}{llll}\text { Salix babylonica } & \text { Arundo donax } & \text { Ficus } \text { sp. } & \text { Ailanthus altissima } \\ \text { Nicotiana } \text { sp. } & \text { Robinia pseudo-acacia } & \text { Ceratonia siliqua } & \text { Platanus } x \text { hispanica } \\ \text { Castanea sativa } & \text { Frutales } & \end{array}$

1) El índice no es aplicable en las zonas más altas de las cuencas en las que no existe de forma natural vegetación arbórea.

2) En las zonas áridas y semiáridas y en las ramblas, se entiende que se contemplan los arbustos con porte arbóreo como los árboles que se consideran en esta hoja de campo.

(Para este ultimo caso, ver la aplicación del índice realizado en Murcia y publicado en Tecnología del Agua) 